\title{
Correction: Cushioned-Density Gradient Ultracentrifugation (C-DGUC) improves the isolation efficiency of extracellular vesicles
}

\section{Phat Duong, Allen Chung, Laura Bouchareychas, Robert L. Raffai}

In Fig 5, panel E erroneously displays the histograms shown in panels B, C, and D. Please see the correct Fig 5 here.

f open Access

Citation: Duong P, Chung A, Bouchareychas L, Raffai RL (2020) Correction: Cushioned-Density Gradient Ultracentrifugation (C-DGUC) improves the isolation efficiency of extracellular vesicles. PLoS ONE 15(7): e0236914. https://doi.org/ 10.1371/journal.pone.0236914

Published: July 23, 2020

Copyright: @ 2020 Duong et al. This is an open access article distributed under the terms of the Creative Commons Attribution License, which permits unrestricted use, distribution, and reproduction in any medium, provided the original author and source are credited. 


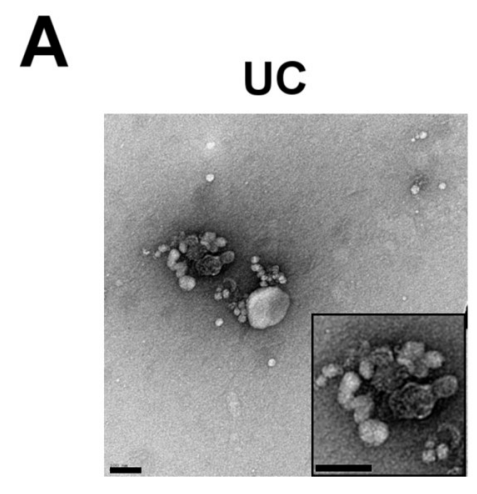

B

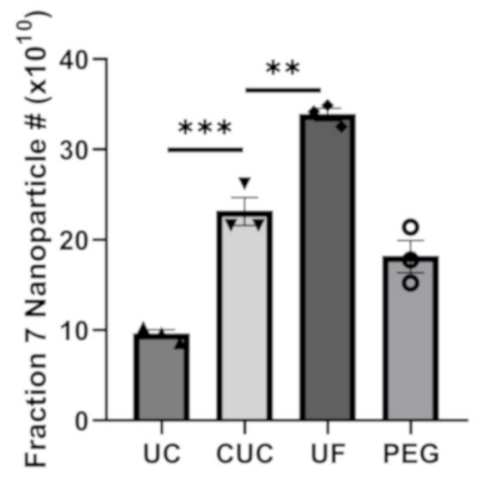

E

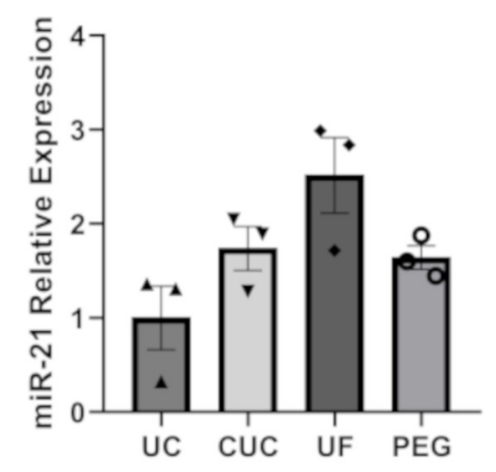

$\mathbf{F}$

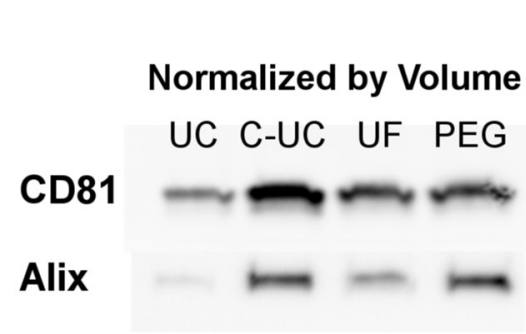

C-UC

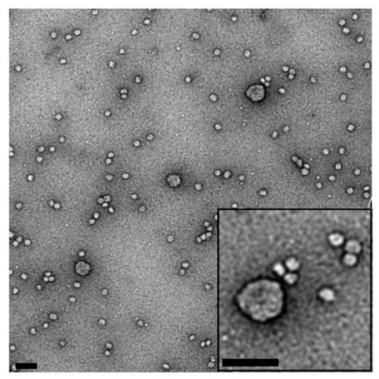

C

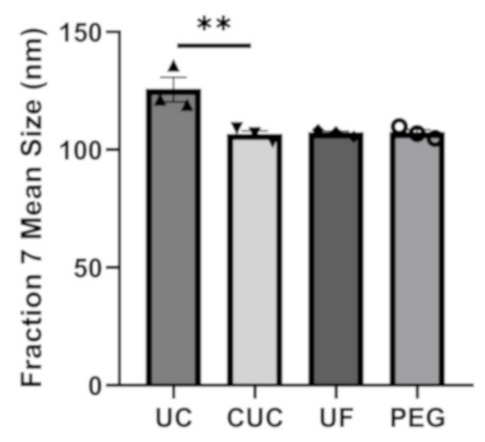

miR-146a

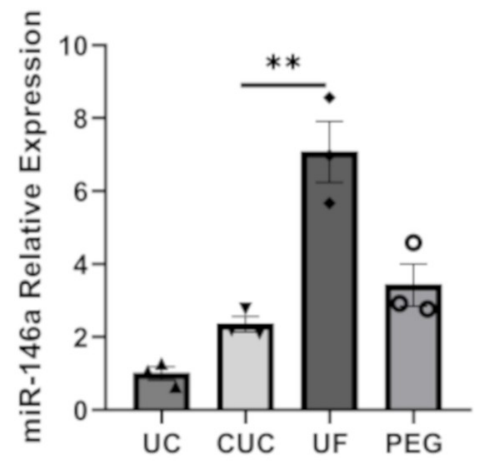

PEG

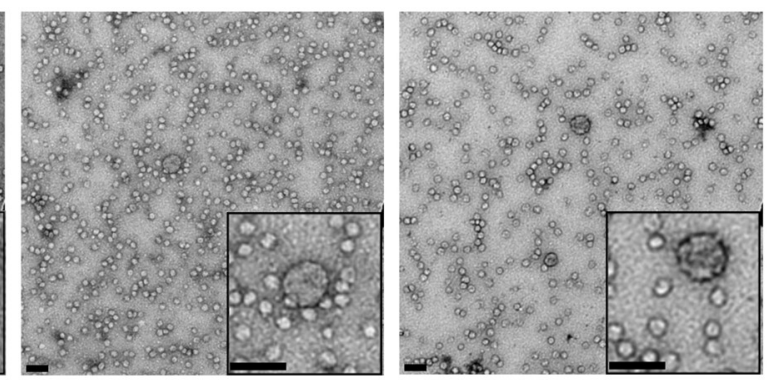

D

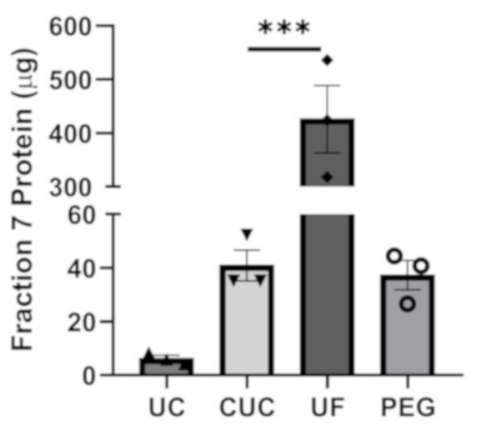

miR-16
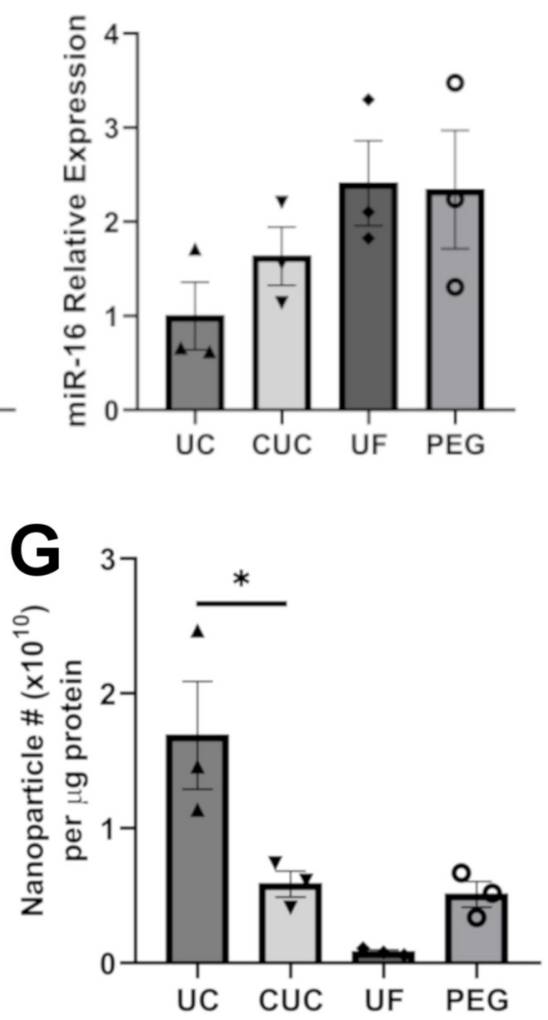


\section{PLOS ONE}

Fig 5. Nanoparticle, protein and RNA analysis of the EV containing fraction. Electron Microscopy of EVs from fraction 7 isolated using different methods with both scale bars representing $100 \mathrm{~nm}$ (A). Nanoparticles in fraction 7 isolated using different methods were enumerated (B) and sized (C) by NTA. Protein mass was quantified by Qubit assay (D). An equal volume ( $200 \mu \mathrm{L})$ was taken from fraction 7 for miRNA analysis. Levels of microRNAs miR-21, miR-146a and miR-16 were measured relative to the synthetic spike-in UniSp2 by qPCR (E). An equal volume $(37.5 \mu \mathrm{L})$ and number $\left(3 \times 10^{9}\right.$ nanoparticles) from fraction 7 of all four methods were taken and assessed for CD81 and ALIX by western blot. Representative blot images are shown (F). The ratio of nanoparticles count to $\mu \mathrm{g}$ protein was plotted as a relative measurement of purity (G). For statistical analysis, a 1-way ANOVA followed with Dunnett's multiple comparison test was used, C-UC served as the control group. Data are expressed as mean \pm SEM from three experiments, ${ }^{*} \mathrm{P}<0.05 ;{ }^{* *} \mathrm{P}<0.01 ;{ }^{* * *} \mathrm{P}<0.001$.

https://doi.org/10.1371/journal.pone.0236914.g001

\section{Reference}

1. Duong P, Chung A, Bouchareychas L, Raffai RL (2019) Cushioned-Density Gradient Ultracentrifugation (C-DGUC) improves the isolation efficiency of extracellular vesicles. PLoS ONE 14(4): e0215324. https://doi.org/10.1371/journal.pone.0215324 PMID: 30973950 